\title{
Effect of smart textiles metamaterials on electromagnetic performance for wireless body area network systems
}

Textile Research Journal

$X X(X): 1-5$

.c The Author(s) 2018

Reprints and permission:

sagepub.co.uk/journalsPermissions.nav

DOI: 10.1177/ToBeAssigned

www.sagepub.com/

(\$)SAGE

\begin{abstract}
In this work, the utilization of different textile materials for manufacturing of metamaterial with the aim of controlling the signal propagation on smart textile applications is investigated. The performance of composite structures of embroidered yarn conductor transmission lines loaded with split-ring resonator geometries in felt and cotton substrates are reported. The proposed structure allows propagating or filtering the transmitted signal in the microwave frequency range. The experimental results exhibit a rejection band between 1.3 and $2.6 \mathrm{GHz}$ for felt substrate and between 1.6 and $2.6 \mathrm{GHz}$ for cotton substrate with stop-band levels lower than $-20 \mathrm{~dB}$. The presented e-textile structures are designed, electromagnetically simulated and measured. The measured results are in good agreement with 3D electromagnetic simulations. The effect of bending of the e-textiles for realistic scenario is also studied. The experimental results show that by changing the radius of bending from $10 \mathrm{~mm}$ to $65 \mathrm{~mm}$, the resonance frequency is shifted up $290 \mathrm{MHz}$ and $144 \mathrm{MHz}$ for cotton and felt substrates, respectively.
\end{abstract}

\section{Keywords}

E-textile, Metamaterial, Split-ring resonator (SRR), Electromagnetic.

\section{Introduction}

Electronic textiles (e-textiles) are a key component of the smart textile field for developing new wearable applications for wireless body area network (WBAN). Such applications are being deployed for sports, fitness, medical, health care and fashion industry sectors ${ }^{1,2}$. For instance, wearable antennas have been recently developed in order to improve the integration of the radio frequency systems on the garments and to allow radio communication ${ }^{3,4}$. On the other hand, metamaterials (MTMs) have attracted significant attention by researchers since the beginning of the 21 st century ${ }^{5}$. These artificial structures are usually designed to obtain controllable and inaccessible electromagnetic (EM) properties not found among natural materials. MTM transmission lines benefit from those properties and they can control the behavior of the guided signals up to a certain level. The microstrip is a typical transmission line used to transmit radio frequency signals and it is commonly fabricated using printed circuit board (PCB) technology. It consists of a strip conductor and a ground back-plane, separated by a dielectric. Its main advantages are a high compatibility with active devices and an excellent balance between cost, size and characteristic impedance control. Its main drawbacks are a low power handling capacity and high losses at high radio frequencies. The first feature could match properly with wearable applications, because a low power is required for safety reasons. Concerning losses, this fact is unavoidable but, in any case, textile substrate losses are intrinsically high in comparison with PCB standard materials. The way of reducing the overall losses of the wearable system is to reduce the loss tangent of the selected fabrics and to use high quality conductive yarns. Different kinds of metamaterial resonators have been investigated to achieve selective frequency responses. Among them, split ring resonator (SRR) is a widely proposed magnetic resonant structure ${ }^{5-9}$. As can be observed from Fig. 1, the SRR structure consists of a ring with a gap, which corresponds to an equivalent inductance $(\mathrm{L})$ and capacitance $(\mathrm{C})$, thus generating an equivalent LC tank. Since we can control and optimize the design of microstrip components by using SRRs, it is theoretically feasible to implement such structures in textile substrates in order to optimize the performance of wearable or e-textile devices, whose main requirements are flexibility, lightweight, low-profile and compactness. 


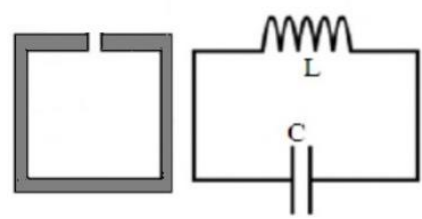

Figure 1. Split ring resonator and its equivalent circuit model.

Embroidery machines enhance the integration of e-textile substrates, because of the repeatability of digital layout patterns. Therefore, mass production of garments and customized designs in terms of thread distribution with a resolution in the order of $<1 \mathrm{~mm}$ can be achieved ${ }^{10}$. Textile MTMs have been directly reported in literature for antenna applications ${ }^{11}$. Textiles have also been used in composite polymer fiber fabrics ${ }^{12}$, and copper split- ring resonators have been used as narrow-band solution to reduce the electromagnetic radiation ${ }^{13}$ and for passive UHF RFID tags ${ }^{14}$. In addition, textile substrates have been used in wearable antenna applied to wireless body area network ${ }^{15-19}$. However, wearable e-textiles have some limitations that must be addressed. One of them is the effect of the electrical impact of the human body, since biological tissues make up a lossy medium. In addition, the body shape changes the electrical performance of the prototypes due to bending and this issue must be addressed. The impact of the e-textile on the body is usually taken into account by means of the specific absorption rate (SAR). Nevertheless, in full grounded structures such as microstrip, this effect could be neglected. Another current limitation of the e-textiles corresponds to washability ${ }^{20-21}$ and durability of the samples, and work is in progress by several research groups to address this issue.

In this work the effect of MTM based on embroidered transmission line loaded with SRR on felt and cotton substrates are analyzed, modeled and measured. In particular, the filtering effect of the textile SRRs is studied. The main idea of the design is to implement embroidered SRR particles in order to filter and control the signal propagation in the ultra-high frequency (UHF) range along the e-textile. Adjustment of the geometrical positioning of the geometrical pattern threads allows achieving tunable resonance frequencies. The main advantage of these structures in comparison with regular transmission line filters is the intrinsic reduction of the dimensions of the filter due to the subwavelength particles used (SRR). Moreover, since we are working with embroidery patterns it is necessary to achieve simple geometries and this is the case of these kind of structures. The proposed prototypes have been simulated by means of the commercial full 3D electromagnetic CST Microwave Studio 2018 software. The proposed prototypes have been subsequently fabricated and analyzed in bandwidths between 1.2-3 GHz in a free space environment. For the measurement of the insertion losses $\left(\mathrm{S}_{21}\right)$ and return losses $\left(\mathrm{S}_{11}\right)$, a N9916A FieldFox (Keysight) vector network analyzer, has been used. The results of the proposed designs show that textile MTMs can be efficiently applied in filtering and controlling the propagation of signals operating in the WBAN relevant frequencies. Among other applications, the presented e-textiles can be considered in the development of wearable sensors, and RFID tags.

The main novelty of the work is to combine full embroidered e-textile designs including metamaterial particles by achieving significant frequency rejection bands in e-textiles. Moreover, the bending effects have been addressed in order to evaluate the impact in several realistic body shape scenarios.

\section{E-textile Metamaterial design and equivalent circuit model}

There are several structure geometries available for SRR such as square, omega shaped, U-shaped, etc. In this work, square shaped SRR have been selected in order to enhance the coupling between the host line and the resonator itself. In addition, this geometry involves a better optimization process in terms of design and simulation. The structure of the proposed design and its relevant dimensions are depicted in Fig. 2(a).

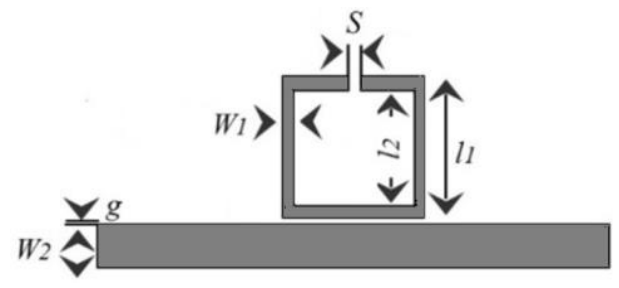

(a)

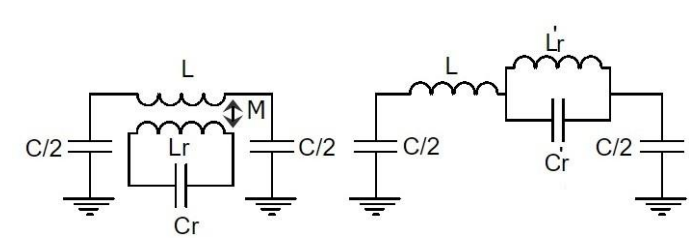

(b)

(c)

Figure 2. (a) Layout of the SRR loaded transmission lines with the relevant dimensions: $11=25 \mathrm{~mm}, 12=22 \mathrm{~mm}, \mathrm{~g}=60 \mu \mathrm{m}, \mathrm{s}=0.18 \mathrm{~mm}$, w1=1.5 $\mathrm{mm}, \mathrm{w} 2=3.9 \mathrm{~mm}$ (b) Lumped element equivalent $\Pi$-circuit model considering magnetic coupling between line and SRR. (c) Simplified circuit results after transformation of the series branch ${ }^{22}$.

At the SRR resonance frequency, the LC tank behavior is exhibited and the guided signal is coupled to the SRR. Therefore, a stop-band effect arises and it is possible to filter the signal propagation by controlling the SRR dimensions and geometry. This effect is reflected in the lumped element equivalent circuit model, depicted in Fig. 2(b). The equivalent circuit model of the series branch can be simplified as depicted in Fig. 2(c).

After a tuning design process with the electromagnetic simulator, the dimensions of the proposed design are set as follows: length of the outer ring $11=25 \mathrm{~mm}$, length of the inner ring $12=22 \mathrm{~mm}$, host line-SRR separation $\mathrm{g}=60 \mu \mathrm{m}$, gap of the SRR 
$\mathrm{s}=0.18 \mathrm{~mm}$, SRR width $\mathrm{W} 1=1.5 \mathrm{~mm}$ and transmission line width $\mathrm{W} 2=3.9 \mathrm{~mm}$. The conventional transmission line has been designed with a $50 \Omega$ characteristic impedance. The transmission line is represented by a $\Pi$ circuit model and the magnetic wall concept was applied in that model, where the $\mathrm{L}$ and $\mathrm{C}$ are the line inductance and capacitance, respectively, of the unit cell; $\mathrm{Lr}$ and $\mathrm{Cr}$ are the inductance and capacitance of the SRR. Finally, $\mathrm{M}$ accounts for the magnetic coupling between the line and the ring. To extract the parameters, the coupling between the host line and SRR has been taking into account and the system has been modeled by means of the commercial Keysight Advanced Design System 2018 software. The electrical parameters have been extracted by means of the method reported in ${ }^{23}$.

\section{Fabric Substrates}

In order to implement the prototype under test (PUT), two different substrate textiles with completely different physical structures have been considered. Firstly, a felt substrate with dielectric constant $\varepsilon_{r}=1.2$, thickness $h_{\text {felt }}=1 \mathrm{~mm}$ and loss tangent $\delta=0.0013$ has been studied. Secondly, a cotton substrate with dielectric constant $\varepsilon r=1.9$, thickness $\mathrm{h}_{\text {cotton }}=0.4 \mathrm{~mm}$ and loss tangent $\delta=0.058$ has been used. As a first step, the parameters of both fabrics (felt and cotton) were designed at a $50 \Omega$ characteristic impedance. The design simulation results demonstrate that the $50 \Omega$ transmission line presents conductive yarn width of $3.9 \mathrm{~mm}$ for felt and $1.3 \mathrm{~mm}$ for cotton, respectively. In order to compare the performance of the two substrates (felt and cotton) in the same geometrical conditions, that is, with the same width for the transmission conductive yarn line (3.9 $\mathrm{mm})$ and the same width of the SRR $(1.5 \mathrm{~mm})$, the two substrates were simulated again. In this case, to achive the same 50 ohm condition for cotton, a higher thickness was required. Therefore, an extra cotton layer has been added by means of commercial adhesive and the overall thickness of the cotton prototype corresponds to $\mathrm{h}_{\text {cotton }}=0.8 \mathrm{~mm}$. In addition, the extra layer prevents a potential short circuit between the host transmission line and the ground plane. Both fabrics are commonly used in the fashion and clothing industry. Table 1 details the main physical characteristics of the two studied fabrics ${ }^{24}$. Fig. 3 and Fig. 4 show the appearance of each of the fabric faces by means of a scanning electron microscope (SEM) micrograph.

Table 1: Main physical properties of the fabrics studied

\begin{tabular}{ll}
\hline Ref & Cotton substrate \\
\hline Composition & Co $100 \%$ \\
Structure & Woven fabric \\
Weight $[\mathrm{g} / \mathrm{m} 2]$ & 204 \\
Structure description & $3 \mathrm{e} 1 \mathrm{~b} 1.3^{* *}$ \\
\hline * Polyester & \\
** Weave fabric specification & \\
&
\end{tabular}

(a)

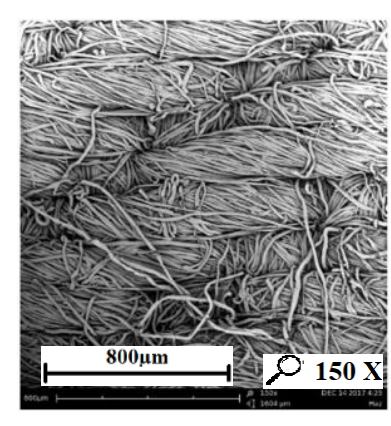

(b)

Figure 3. Appearance of the studied fabrics, scanning electron micrograph (a) Face A (light sarge) Cotton. Warp direction $\uparrow$ (b) Face B (heavy sarge) Cotton. Warp direction $\rightarrow$

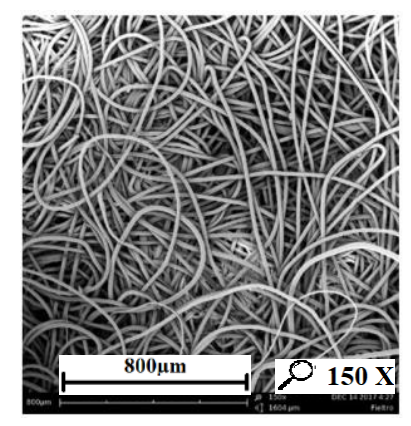

(a)

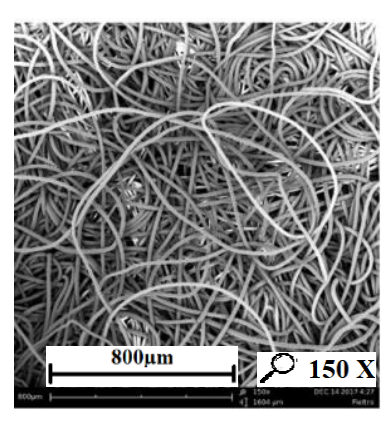

(b)

Figure 4. Appearance of the studied fabrics, scanning electron micrograph (a) Face A (light sarge) Felt. Warp direction $\uparrow$ (b) Face B (heavy sarge) Felt. Warp direction $\rightarrow$ 
A Singer Futura XL-550 embroidery machine has been used to manufacture the prototype. The used stitch type corresponds to the ISO 4915:1991 301 standards. For the PUTs, the selected conductor yarn corresponds to a commercial Shieldex Plated Nylon 66 Yarn 117/17 dtex 2- ply and it is composed by 99\% pure silver plated nylon yarn 140/17 dtex with a linear resistance $<30 \Omega / \mathrm{cm}$.

The conductive thread is relatively thick compared to the conventional embroidery thread, due to the mechanical restrictions of the embroidery machine. In order to optimize the fabrication, process the conductive thread has been used in the bobbin of the embroidery machine whereas a conventional embroidery yarn has been considered for the upper thread. A certain degree of tension control in the upper thread is carried out in order to increase the accuracy of the stitching geometries and patterns.

The proposed design has been embroidered with satin pattern with $60 \%$ and $40 \%$ density for felt and cotton, respectively. The stitch spacing corresponds to the distance between two needle penetrations on the same side of a column. The density determines the gap between stitches. For narrow columns, stitches are tight, thus requiring fewer stitches to cover the fabric. In areas with very narrow columns, less dense stitches are required because too many needle penetrations can damage the textile sample. The homogeneous layout is converted to a stitch pattern by using the Digitizer Ex software for fabrication process. This software package is used to create the stitch pattern, which is then exported to the embroidery machine and stitched. The effect of the stitch direction and stitch density has been extensively studied and described by ${ }^{25}$.

The ground plane for both prototypes have been chosen as a homogeneous uniform commercial WE-CF adhesive copper sheet layer (constant thickness $\mathrm{t}=35 \mu \mathrm{m}$ ), for simplicity. The embroidered prototype on felt substrate is depicted in Fig. 5(a).

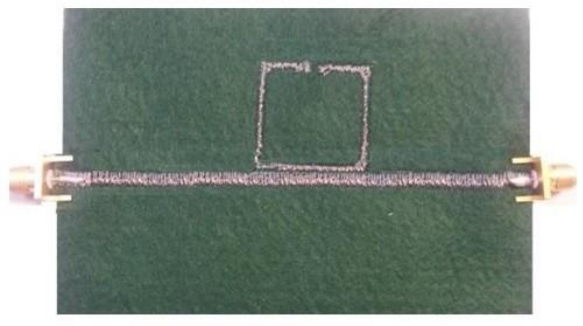

(a)

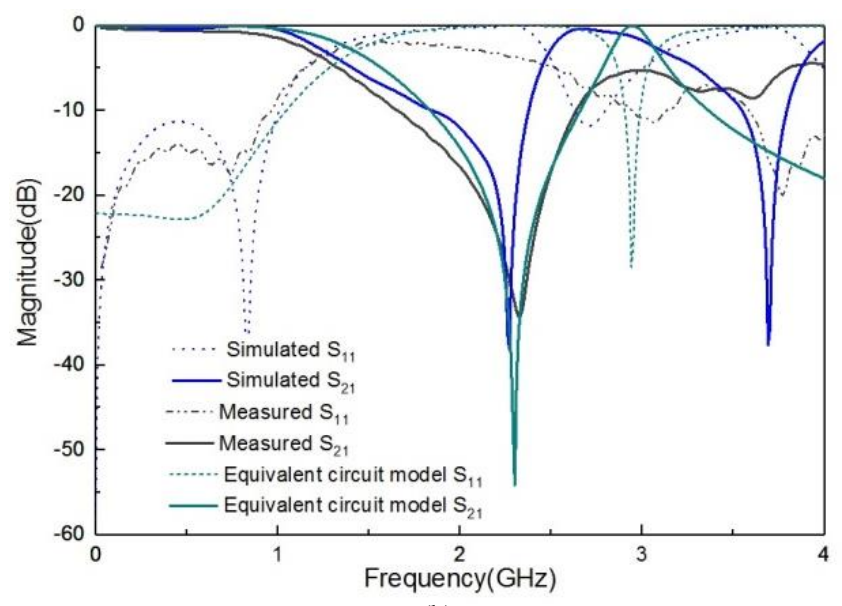

(b)

Figure 5. (a) Photograph of the embroidered transmission line loaded with SRR on felt substrate with satin pattern (60\% density). (b) Sparameter responses of the EM simulation, equivalent circuit model and measurement of the proposed design.

The results of simulated and equivalent circuit model analysis and measured result on felt substrate is shown in Fig. 5(b). The extracted parameters of equivalent circuit model are the following: $\mathrm{L}=6.5 \mathrm{nH}, \mathrm{C}=3 \mathrm{pF}, \mathrm{Cr}=0.3 \mathrm{pF}, \mathrm{Lr}=16 \mathrm{nH}, \mathrm{M}=0.55$ $\mathrm{nH}$. A good agreement between the simulated, equivalent circuit model and the measured results is obtained. As can be observed, the proposed design exhibits a well-defined stop band, defined at $\left|S_{21}\right|_{d B}=3 \mathrm{~dB}$, between 1.3 and $2.6 \mathrm{GHz}$.

The most interesting properties of the SRR is that the orientation and position of its gap with respect to the hosting transmission line has significant influence on the overall performance and also the number and configuration of the SRR can change the behavior of the filter from stop-band to pass-band filter. In this work, the gap of SRR is far from line and the measured return loss oscillates between $-0.5 \mathrm{~dB}$ and $-3.5 \mathrm{~dB}$ and the insertion loss is $\left|S_{21}\right|_{d B}=30 \mathrm{~dB}$ at $2.3 \mathrm{GHz}$. On the other hand, a reasonable level of losses can be achieved at the band-pass, for instance $-4 \mathrm{~dB}$ at $1.6 \mathrm{GHz}$. Therefore, a high degree of attenuation can be achieved with single embroidery SRR.
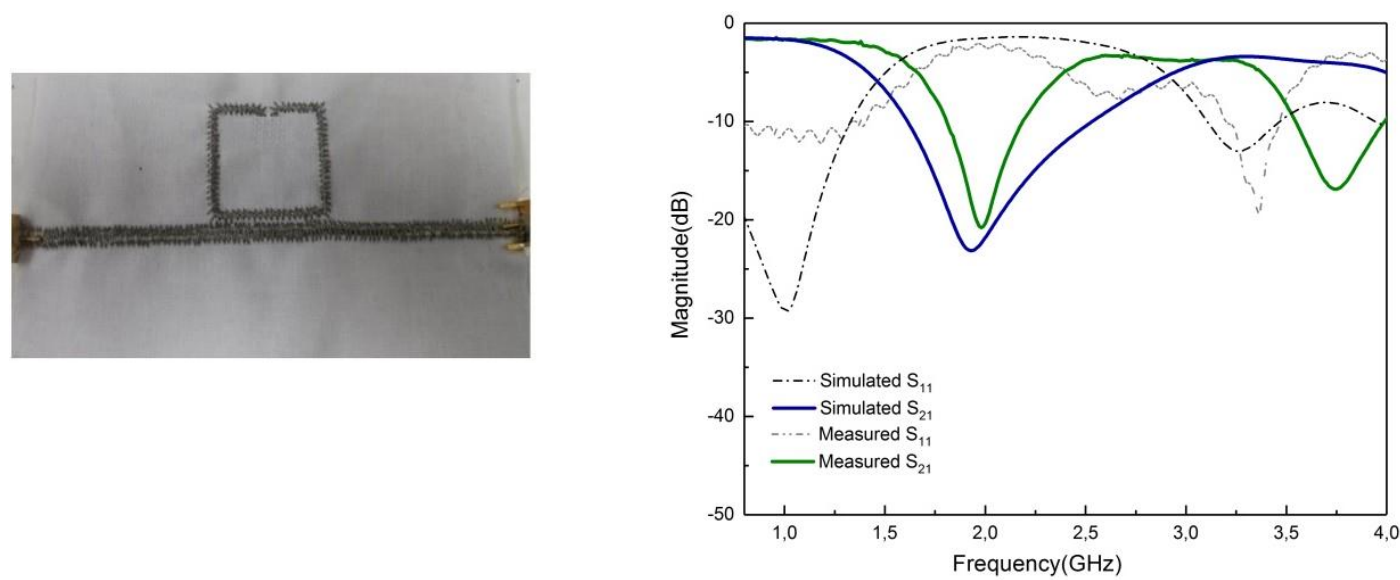
Figure 6. (a) Photograph of the embroidered transmission line loaded with SRR on cotton substrate with pattern satin (40\% density). (b) Sparameter responses of the EM simulation and measurement of the proposed design.

Fig. 6(a) shows the embroidered proposed design on cotton substrate. The comparison of simulation and measurement result of proposed design on cotton substrate is shown in Fig. 6(b). There is good agreement between the simulated and the measured results. The proposed design provides stop band between 1.6 and $2.6 \mathrm{GHz}$. The measured return loss in the rejection band oscillates between $-0.5 \mathrm{~dB}$ and $-2.5 \mathrm{~dB}$ and in the band-pass $-3.5 \mathrm{~dB}$, indeed, the insertion loss is $\left|S_{21}\right|_{d B}=20$ $\mathrm{dB}$ at the resonance frequency, $2 \mathrm{GHz}$.

The electromagnetic properties of the two substrates are listed in Table 2. The reported experimental values demonstrate that MTM-SRR composite textile materials provide a significant frequency control of the propagated signals for practical wearable applications.

Table 2. Comparison measurement of two substrate electromagnetic properties

\begin{tabular}{lll}
\hline Parameter & Cotton substrate & Felt substrate \\
\hline Band width (@-3 dB) & $1 \mathrm{GHz}$ & $1.3 \mathrm{GHz}$ \\
Return Losses (@ stop band) & $2.5 \mathrm{~dB}$ & $3.5 \mathrm{~dB}$ \\
Losses (@ band-pass) & $3.5 \mathrm{~dB}$ & $4 \mathrm{~dB}$ \\
Insertion loss(@ stop band & $\geq 20 \mathrm{~dB}$ & $\geq 30 \mathrm{~dB}$ \\
Resonance frequency & $2 \mathrm{GHz}$ & $2.3 \mathrm{GHz}$ \\
\hline
\end{tabular}

The experimental characteristic impedance for the felt substrate sample is $50 \Omega$, whereas for the cotton substrate sample corresponds to $42 \Omega$. The slight discrepancy for the cotton case with regard to the simulated $50 \Omega$ is due to the difficulties of measuring the two layer case, including inhomogeneous thickness of the adhesive that was used for sticking the cotton layers. The main advantage of the felt substrate is that it provides good impedance matching and low losses compared to cotton. Also, by comparing Figs. 5(b) and 6(b) it is observed that, the insertion loss in felt substrate are better than in the cotton case. The reason is that better accuracy is expected for felt due to have low embroidery tension. Thus, to achieve high geometrical accuracy for felt, we have increased the embroidery density up to $60 \%$ to boost surface conductivity.

\section{Effects of bending}

In wearable systems, it is very difficult to keep the substrate on flat configuration all the time, especially when the prototype is made of textile materials and it is frequently bent due to human body morphology and movements. Therefore, it is necessary to investigate the prototypes performance characteristics under bending conditions. The $\mathrm{S}_{21}$ parameters of e-textile MTM-SRR under different bending have been measured.

It is observed that due to bending, the equivalent length of the proposed design gets changed and, hence, there are deviations in the resonance frequency. The more the prototype is bent, the more the resonant length gets reduced and so the resonant frequency gets shifted up. This fact becomes evident from the experimental observations, as shown in Fig.7 (a) and Fig. 7(b). By changing the radius of bending from $10 \mathrm{~mm}$ to $65 \mathrm{~mm}$, the resonant frequency is shifted up $290 \mathrm{MHz}$ for the cotton substrate and $144 \mathrm{MHz}$ for felt case. From the S-parameters measured results in Fig.7 (a), two high $\mathrm{S}_{21}$ peaks $-27 \mathrm{~dB}$ and $25 \mathrm{~dB}$ are observed at radius of $10 \mathrm{~mm}$ and $65 \mathrm{~mm}$ respectively. As can be seen in Fig. 7(b), there are one minimum $\mathrm{S}_{21}$ peak $(-15 \mathrm{~dB}$ at $2.25 \mathrm{GHz})$ with regard to a radius of $10 \mathrm{~mm}$ and one maximum $\mathrm{S}_{21}$ peak $(-37.5 \mathrm{~dB}$ at $2.46 \mathrm{GHz})$ corresponding to a radius of $30 \mathrm{~mm}$.

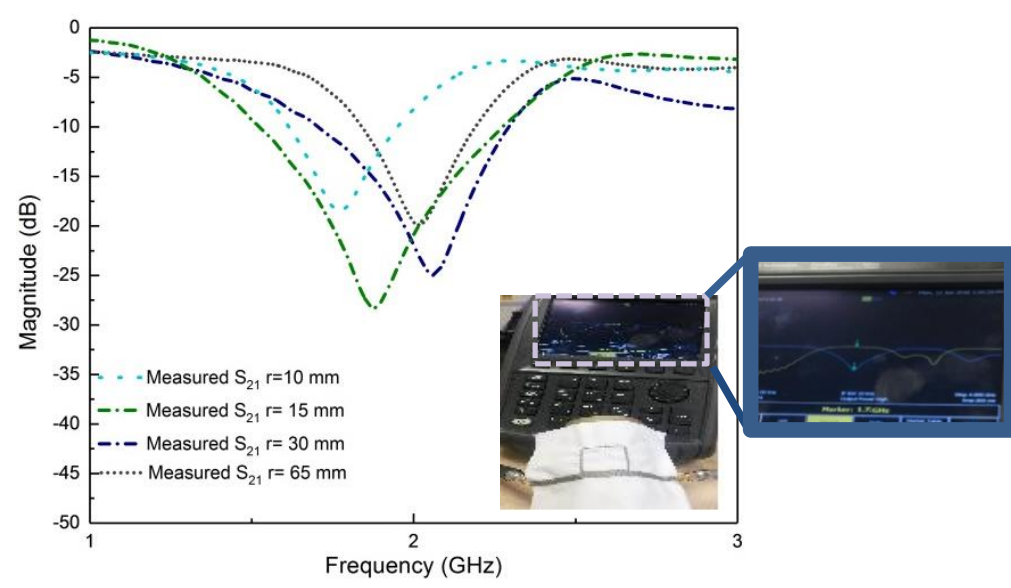

(a)

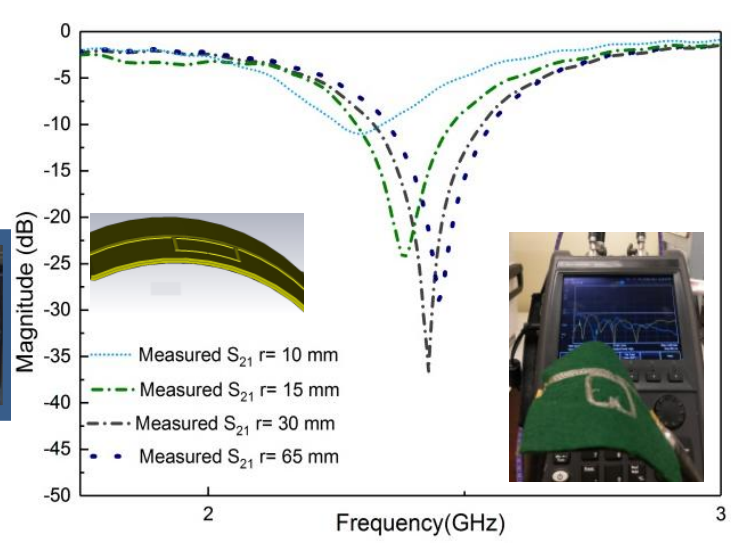

(b) 
Figure 7 S21 effect of bending with different radius: $10 \mathrm{~mm}, 15 \mathrm{~mm}, 30 \mathrm{~mm}$ and $65 \mathrm{~mm}$ on (a) Cotton substrate (b) Felt substrate.

\section{Conclusion}

In this work, the utilization of different fabric materials for the implementation of e-textile metamaterial transmission lines is investigated with the aim of controlling the signal propagation on smart textile applications. The proposed design is a fully embroidered conductive thread transmission line loaded with conductive yarn SRR on felt and cotton substrates. A significant agreement is achieved for the electromagnetic layout simulations and the experimental results. The measurement results exhibit a well-defined stop-band of $1.3 \mathrm{GHz}$ on felt and $1 \mathrm{GHz}$ on cotton substrate with a high level of signal rejection. Also the effects of bending of the manufactured e-textiles have been tested and quantified, obtaining a relatively low impact on the resonance frequency of the proposed designs in terms of typical bending parameters due to conformal values to the human body shape.

\section{Acknowledgements}

This work was supported by the Spanish Government MINECO under project TEC2016-79465-R.

\section{References}

1 S. Schneegass and O. Amft, Smart Textiles. Springer, 2017.

2 A. M. Grancaric, I. Jerkovic, V. Koncar, C. Cochrane, F. M. Kelly, D. Soulat, and X. Legrand, "Conductive polymers for smart textile applications,” Journal of Industrial Textiles, p. 1528083717699368, 2017.

3 L. Yao, X. Wang, F. Xu, M. Jiang, D. Zhou, and Y. Qiu, "Effect of wire space and weaving pattern on performance of microstrip antennas integrated in the three dimensional orthogonal woven composites," Applied Composite Materials, vol. 19, no. 1, pp. 21-30, 2012.

4 F. Xu, L. Yao, D. Zhao, M. Jiang, and Y. Qiu, "Effect of weaving direction of conductive yarns on electromagnetic performance of 3d integrated mi- crostrip antenna," Applied Composite Materials, vol. 20, no. 5, pp. 827-838, 2013.

5 J. B. Pendry, A. J. Holden, D. Robbins, and W. Stewart, "Magnetism from conductors and enhanced nonlinear phenomena," IEEE transactions on microwave theory and techniques, vol. 47, no. 11, pp. 2075-2084, 1999.

6 D. D. Stancil, "Theory of magneto static waves," Springer-Verlag, 1993.

7 V. G. Veselago, “The electrodynamics of substances with simultaneously negative values of s and $\mu$,” Phys. Usp., vol. 10, no. 4, pp. 509-514, 1968. [Online]. Available: https://ufn.ru/en/articles/1968/4/d/

8 R. A. Shelby, D. R. Smith, and S. Schultz, "Experimental verification of a negative index of refraction," science, vol. 292, no. 5514, pp. 77-79, 2001.

9 H. Mosallaei and K. Sarabandi, "Design and modeling of patch antenna printed on magneto-dielectric embedded-circuit meta substrate," IEEE Transactions on Antennas and Propagation, vol. 55, no. 1, pp. 45-52, 2007.

10 A. Tsolis, W. G. Whittow, A. A. Alexandridis, and J. Vardaxoglou, "Embroidery and related manufacturing techniques for wearable antennas: challenges and opportunities," Electronics, vol. 3, no. 2, pp. 314-338, 2014.

11 R. Seager, A. Chauraya, J. Vardaxoglou, and P. De Maagt, "Fabric antennas integrated with metamaterials," in IEEE European Microwave Conference, 2008.

12 M. S. Mirotznik, S. Yarlagadda, R. McCauley, and P. Pa, "Broadband electromagnetic modeling of woven fabric composites," IEEE Transactions on Microwave Theory and Techniques, vol. 60, no. 1, pp. 158-169, 2012.

13 S. Dudzinska, I. Krucinska, and R. Brazis, "Textiles embroidered with split- rings as barriers against microwave radiation,” Fibres \& Textiles in Eastern Europe, vol. 17, no. 1, p. 72, 2009.

14 S. Ma, L. Ukkonen, L. Sydanheimo and T. Bjorninen, "Wearable E-textile split ring passive UHF RFID tag: Body-worn performance evaluation," Asia Pacific Microwave Conference (APMC), pp. 166-168, 2017.

15 I. Gil and R. Fernandez-Garcia, "Embroidery manufacturing techniques for wearable dipole antenna applied to wireless body area network," Textile Research Journal, 2018.

16 C. Hertleer, A. Van Laere, H. Rogier, and L. Van Langenhove, “Influence of relative humidity on textile antenna performance,” Textile Research Journal, vol. 80, no. 2, pp. 177-183, 2010.

17 C. Hertleer, A. Tronquo, H. Rogier, and L. Van Lange hove, "The use of textile materials to design wearable microstrip patch antennas," Textile Research Journal, vol. 78, no. 8, pp. 651-658, 2008.

18 I. Locher and G. Troster, "Screen-printed textile transmission lines," Textile Research Journal, vol. 77, no. 11, pp. 837-842, 2007.

19 M. Karimiyan-Mohammadabadi, M. Dorostkar, F. Shokuohi, M. Shanbeh, and A. Torkan, "Ultra-wideband textile antenna with circular polarization for gps applications and wireless body area networks,” Journal of Industrial Textiles, vol. 46, no. 8, pp. 1684-1697, 2017. 
20 M.L Scarpello, I. Kazani, C. Hertleer, H. Rogier and D.V. Ginste, "Stability and Efficiency of Screen-Printed Wearable and Washable Antennas". IEEE Antennas and Wireless Propagation Letters, vol. 11, pp. 838-421, 2012.

21 M. Toivonen et al., "Impact of Moisture and Washing on the Performance of Embroidered UHF RFID Tags". IEEE Antennas and Wireless Propagation Letters, vol. 12, pp. 1590-1593, 2013.

22 RRA, Syms and Shamonina, E and Kalinin, V and Solymar, L, "A theory of metamaterials based on periodically loaded transmission lines: Interaction between magneto inductive and electromagnetic waves” Journal of Applied Physics, vol. 97, no. 6, 2005.

23 J.-S. G. Hong and M. J. Lancaster, Microstrip filters for RF/microwave applications. John Wiley \& Sons, 2004, vol. 167.

24 Y. Ouyang and W. J. Chappell, "High frequency properties of electro- textiles for wearable antenna applications," IEEE Transactions on Antennas and Propagation, vol. 56, no. 2, pp. 381-389, 2008.

25 R. Seager, S. Zhang, A. Chauraya, W. Whittow, Y. Vardaxoglou, T. Acti, and T. Dias, "Effect of the fabrication parameters on the performance of embroidered antennas," IET Microwaves, Antennas \& Propagation, vol. 7, no. 14, pp. 1174-1181, 2013. 